\title{
PECULIARITIES OF THE INTERNAL PHOTOMETRICAL CALIBRATION METHOD USING DENSITY STELLAR PROFILES
}

\author{
H. MARKOV \\ Rozhen National Astronomical Observatory \\ BG-4700 Smoljan, P.O. Box 136 \\ Bulgaria
}

On photographic plates taken for the purposes of classical stellar photometry (using a standard stellar sequence) there usually is no external photometrical calibration scale, neither a wedge nor spots; and hence the transition to relative intensity is a problem. The construction of a characteristic curve $\mathrm{D}(\mathrm{I})$ (hereafter $\mathrm{CHC}$ ) by combining density profiles of several standard stars is a perfect solution of this task (Agnelli et al. 1979; de Vaucouleurs 1984). We have developed this method in order to obtain internal photometric calibrations for a large number of plates taken at the $\mathrm{RC}$ focus of the $2 \mathrm{~m}$ telescope (F/8, scale 12.86) at the National Astronomical Observatory (NAO); these do not have any photometrical calibration. Our long term project provides UBV stellar photometry in the central regions of some globular clusters - M5, M10, M12, M56, M71, for which we have good collections of short exposure plates. The task also requires a background determination which can be done precisely only in relative intensities. We apply the method using Arp's UBV standard sequence in M5 globular cluster. In our investigation we demonstrate some peculiarities of the method:

1) careful image centering and profile adjustment, especially in the case of extended stellar images, will prevent difficulties concerning the CHC's shape;

2) ensuring equal background of star images whose profiles are to be used in a pair is a required condition under which the method can be applied correctly;

3) well-defined reference magnitudes should be used for the method application because of its sensitivity to the stellar magnitude value (Fig. 1), and hence the method can be used directly for stellar photometry;

4) the photographic emulsion is sensitive not only to the intensity but to the spectral distribution of the illuminating light. We thus should expect stars of different colors to affect emulsion in a different manner (Fig. 2). To avoid significant color effects in the instrumental magnitudes we have to use in the pairs stars with close color indices corresponding to the transparency maximum of the system in a given band (Fig. 3).

The whole procedure of applying the method can be computerised. 


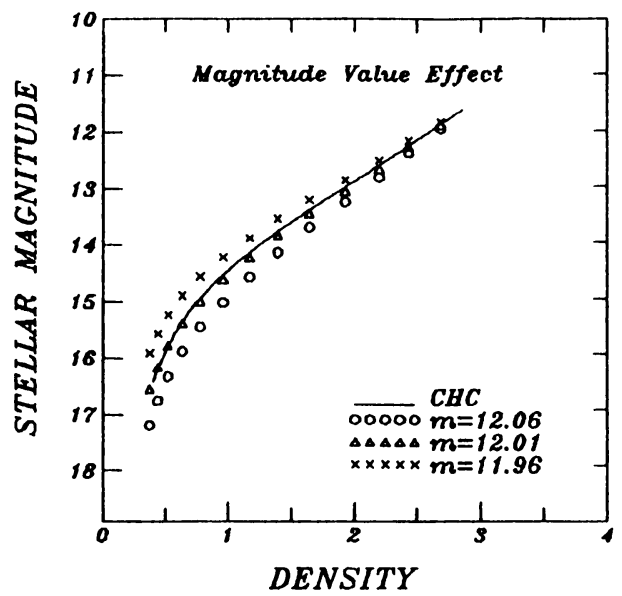

Figure 1. Changing the magnitude value of one of the stars, we obtain single CHCs with different slopes. There is a magnitude value for which a new CHC (open triangles) coincides with an already derived CHC.

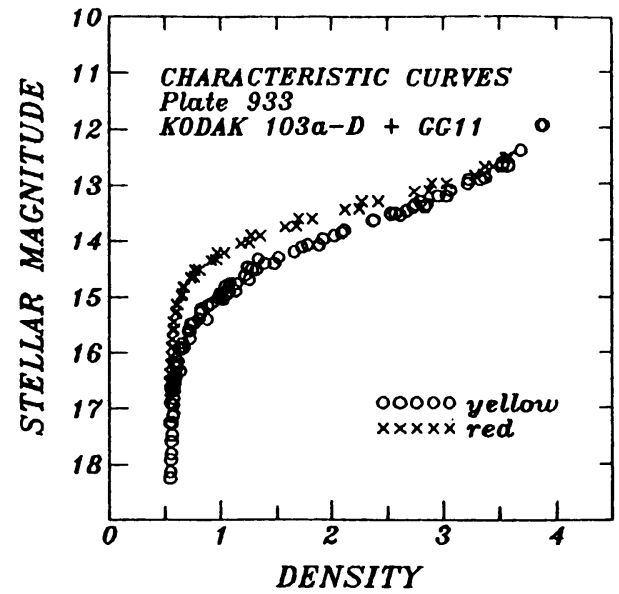

Figure 2. CHCs derived from yellow (open circles) and red (crosses) stars. The difference in the slopes is obvious.

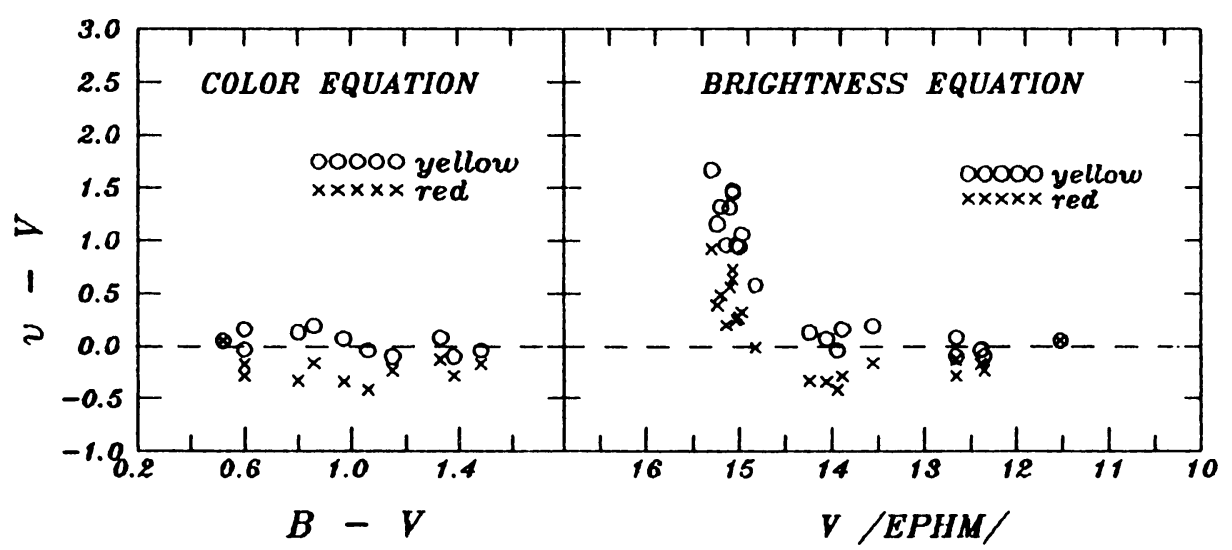

Figure 3. Color equations (left panel) and brightness equations (right panel) obtained when yellow or red stars are used for CHC derivation. Obviously using in the V band a CHC derived with yellow stars we are closer to the reference photometric system.

\section{References}

Agnelli, G., Nanni, D., Pitella, G., Trevese, D. and Vignato, A., 1979. Astron. Astrophys., 77, 45.

de Vaucouleurs, G., 1984. In 'Proc. of the Confer., Astronomy with Schmidt type Telescopes', ed. M. Capaccioli, D. Reidel P. Co., Erice. 